\title{
An accessible electrical impedance tomograph for 3D imaging
}

\section{B.D. Grieve, S. Murphy, A. Burnett-Thompson and T.A. York}

School of Electrical \& Electronic Engineering, University of Manchester, UK

\begin{abstract}
A new electrical tomograph that is designed specifically for low-cost and accessibility is described. It offers a unique environment for three-dimensional (3D) electrical impedance imaging. Using a cross-bar switch, the hardware can support ad hoc measurement strategies and is linked to control software, which integrates the complete process from 3D finite element modelling through to image reconstruction and generation of movie files. Digital signal processing algorithms are used to derive measurements of complex impedance from the measurement of amplitude and phase difference between the driven and received signals. This is achieved by rapid sampling of sinusoidal signals having frequencies between $1.0 \mathrm{~Hz}$ and 1.0 MHz. The instrument can deliver about 100 measurements per second, corresponding to a typical 16-electrode tomography frame, and is targeted at processes with modest dynamics or for laboratory development of tomographic applications and algorithms. The latest version (LCT2) is described here and uses a USB2 link to provide data transfer rates up to 20 MBytes per second. Sinusoidal excitation signals are derived using a direct digital synthesis chip. Voltage measurements are digitized to 16-bit accuracy and the standard system can accommodate 64 electrodes. The software embraces the EIDORS-3D soft-field reconstruction algorithms and the resulting impedance imaging capability of the device and characterization of the signals are described.
\end{abstract}

Key words: 3D image reconstruction; digital signal processing; electrical impedance tomography.

\section{Introduction}

The basic aim of modern tomography is to determine the distribution of materials in some region of interest by obtaining a set of measurements using sensors that are

Address for correspondence: T.A. York, School of Electrical \& Electronic Engineering, University of Manchester, UK. E-mail: t.a.york@umist.ac.uk

Figures 6 and 8-19 appear in colour online: http://tim.sagepub.com

(C) 2010 The Institute of Measurement and Control

$10.1177 / 0142331208100108$ 
distributed around the periphery. For instance, in medical applications the contrasting materials may be normal and cancerous tissue or for industrial applications the materials could be oil and gas in a pipeline. Tomographic measurements are generally non-intrusive, typically penetrating the wall of a vessel but not entering the medium, and in many cases they can be non-invasive, such that the sensors are located on the outside of the bounding wall. Each measurement is affected, to a greater or lesser degree, by the location of materials in the region of interest. Typically, a source of energy is imposed on the vessel from one orientation and a number of measurements are taken by distributed sensors to create a projection of data. The source is then moved to provide another projection and so on around the vessel until a frame of data is accumulated. Usually the frame of data is translated, in software, into a cross-sectional image representing the distribution of materials. Tomography has enjoyed considerable success in medical applications, for instance in the identification of tumours, particularly using $X$-rays as a source of energy to identify contrasting material density from the attenuation of the transmitted signal. More recently, magnetic resonance and electrical excitation tomographies, amongst others, have emerged offering alternative capabilities that can be usefully exploited, such as an ability to distinguish fine structures within live biological specimens (Bottomley et al., 1986) or compatibility with hazardous production environments (Davidson et al., 2004).

It should be noted that, strictly speaking, 'tomography' refers to the reconstruction of a two-dimensional (2D) image. The origin of the term is well documented, coming from ' $\tau \mathrm{o} \mu \sigma^{\prime}$ ', the Greek word for slice. However, reference to a Greek-English dictionary suggests that ' $\tau$ o $\mu \mathrm{o} \sigma$ ' actually means 'volume'. Learned colleagues tell us that this seeming contradiction is explained by considering 'volume' as, for instance, 'Volume 1 of the Encyclopaedia Britannica'. In other words, a slice of the set. This discussion is relevant here where we discuss the reconstruction of three-dimensional (3D) images of volumes. The use of the word 'tomography' in such cases has attracted some debate and we suggest that the seemingly erroneous use of the word might be forgiven, or perhaps even adapted, considering the above.

Over the last 15 years, electrical tomography (York, 2005) has grown into a powerful technique for interrogating industrial processes. Many applications have been explored and these are comprehensively reported in the literature. For instance, these include: the application of electrical resistance tomography to pharmaceutical processes (Ricard et al., 2003); imaging the flow profile of molten steel through a submerged pouring nozzle (Ma et al., 2003); the application of electrical resistance tomography to a large volume production pressure filter (Grieve et al., 2003); flow analysis (Hunt et al., 2003); and electrical capacitance tomography for measurement of gas-solids flow characteristics in a pneumatic conveying systems (Jaworski and Dyakowski, 2001). At the present time, systems for industrial applications focus on one component of the impedance, conductance, capacitance or inductance. This is largely because of the desire for high acquisition rates, which have necessitated bespoke electronics for each component. Acquisition rates up to 1000 frames per 
second have been reported (Wilkinson et al., 2005) and imaging rates up to 100 frames per second. Such performance measures are usually understood to refer to a 'standard' 16-electrode sensor using the adjacent measurement strategy. It should be noted that precedents exist in medical applications for tomographs that measure complex impedance (Hartov et al., 2000). Three start-up companies - Process Tomography Ltd, Industrial Tomography Systems Ltd and Tomoflow Ltd - have grown out of the research undertaken in the UK and instruments for industrial applications are commercially available. Although research into 3D image reconstruction has been reported previously, this capability is not yet fully available in these commercial systems.

In recent years, new application areas have been identified with more modest process dynamics and consequently this places fewer demands on computing requirements. It is also becoming apparent that there is the potential to consider both the real and imaginary components of the impedance, for instance in situations where the medium can change from a continuous conducting phase to a continuous dielectric phase such as in an crude oil pipeline which may contain predominantly water, sand or oil at any one time. Finally, there is a need for low-cost laboratory instruments to support algorithm development and exploratory studies of new applications in which the user requires ready access to the data processing environment. These issues have motivated the University of Manchester and Syngenta to explore the design of a new tomography system, the LCT2, as described below.

\section{LCT system design}

The LCT2 is based on the principle of sequentially multiplexing a signal source and a measurement circuit on to the electrodes of a tomographic sensor under the control of a host PC. A sinusoidal current is delivered to selected electrodes and the resulting voltages around the sensor are amplified and conditioned prior to digitization. The PC is interfaced to the signal conditioning board (SCB) using a USB2link implemented using the Cypress EZ-USB FX2. Using digital signal processing algorithms, amplitude and phase information is recovered from the rapidly sampled current and voltage measurements, and this provides true electrical impedance tomography. A schematic of the latest system (LCT2) is shown in Figure 1. A more detailed view of the SCB and its interface to the software and multiplexer is shown in Figure 2.

The analogue output module, shown in Figure 3, is the same as that used in the earlier LCT1 system (York et al., 2004). A direct digital synthesis (DDS) chip is used to generate sinusoidal excitation waveforms at frequencies from $1.0 \mathrm{~Hz}$ and up to 1.0 $\mathrm{MHz}$ across a range of peak-to-peak amplitudes of $1 \mathrm{mV}$ to $10 \mathrm{~V}$. A programmable gain amplifier (PGA), comprising dual non-inverting amplifiers (AD8021) and a DG409 multiplexer, amplifies the DDS output to enable maximum output voltages of $12 \mathrm{~V}$ peak-peak. The resulting amplified waveform is applied to the electrodes via a highprecision current sensing resistor, which enables the supplied current to be determined. 
This is in contrast to typical electrical tomography systems for process duties, which attempt to deliver accurately controlled currents across the target bandwidth. Previous systems for medical electrical tomography have used a similar approach (Hartov et al., 2000). While the AD8021 exhibits excellent high-frequency properties for use in programmable gain circuits, it is not well suited to reactive loads. The output drive must be able to accommodate a wide range of impedances and

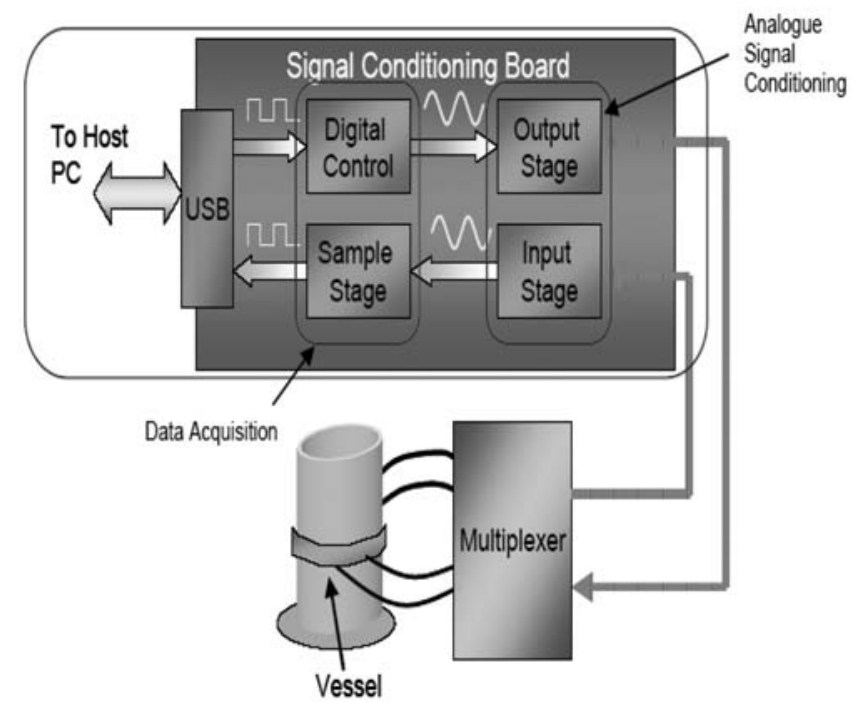

Figure 1 Schematic of LCT2 system

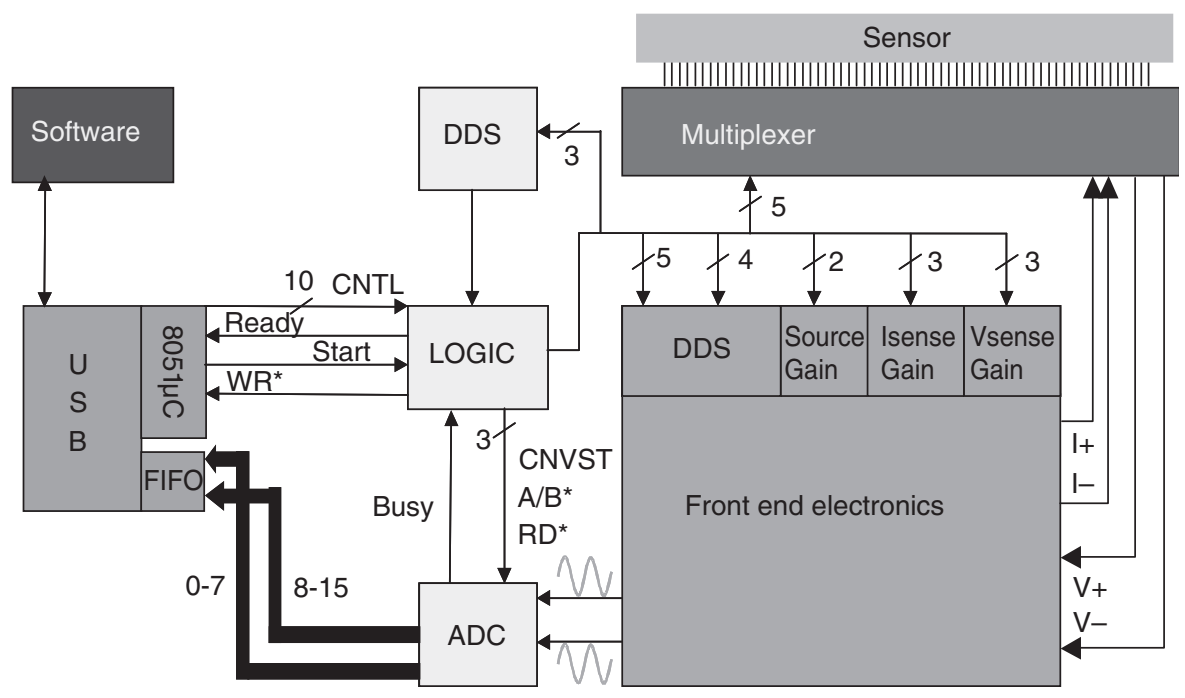

Figure 2 Schematic of signal conditioning board 
signal frequencies. To accomplish this, voltage buffers have been constructed based on the AD817 operational amplifier.

The input stage amplifies the small measured signals, allowing them to be sampled using the full resolution of the 16-bit AD7654 analogue-to-digital converter (ADC). The ADC operates at $250 \mathrm{kHz}$ and therefore, in order to process signals up to $1 \mathrm{MHz}$, both oversampling and undersampling have been employed (Hartov et al., 2000). The input stage is designed to improve on the specification of a commercially available instrumentation amplifier (IA), the INA110 from Texas Instruments. A custom programmable gain IA has been implemented using a fixed gain IA followed by a PGA as shown in Figure 4. The IA allows the gain to be changed between two values using a relay and, combined with the PGA, this cascaded design provides selectivity between eight gains. The resulting instrumentation amplifier has a high input impedance, a gain bandwidth product greater than $100 \mathrm{MHz}$ and a slew rate of $135 \mathrm{~V} / \mu \mathrm{s}$. PSPICE circuit simulations suggest that the constructed IA has a $1-\mathrm{T} \Omega$ input resistance over the desired $1-\mathrm{MHz}$ frequency range with better slew rate than that available in the commercial integrated version.

A schematic diagram of the data acquisition module is shown in Figure 5. It is necessary to measure the output current and the measured input voltages and two-channel simultaneous digitization is desired to preserve phase information. In order to achieve this, the LCT2 uses the AD7654 from Analog Devices. This is a 16-bit, two-channel ADC with a maximum sample rate of $250 \mathrm{ksamples} / \mathrm{s}$ per channel, an analogue bandwidth of $10 \mathrm{MHz}$ and a parallel interface. In order to digitize sinusoidal excitation signals up to

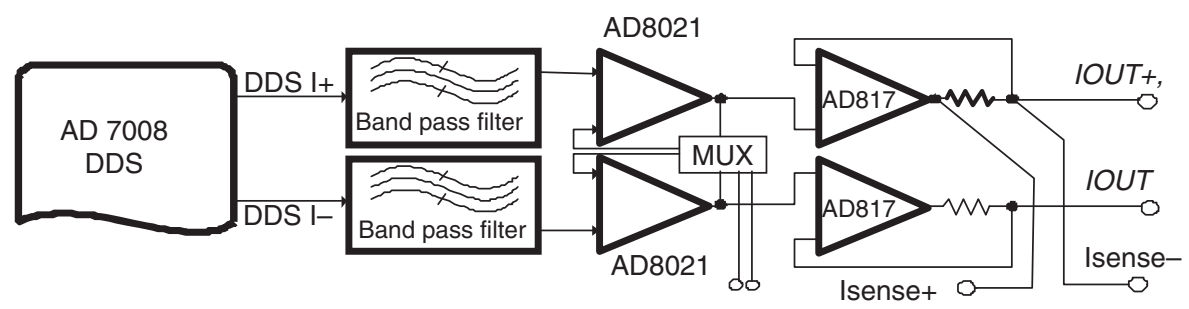

Figure 3 Analogue output module

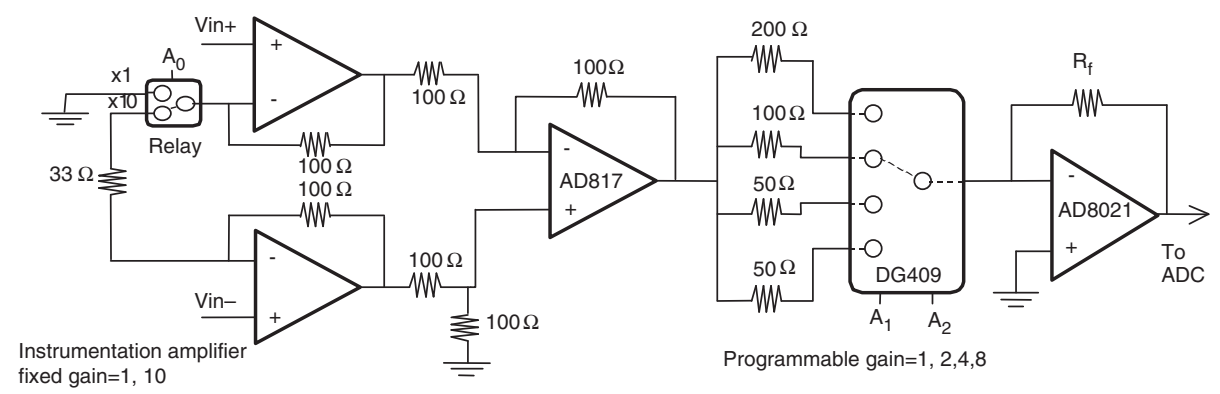

Figure 4 Input stage 
and beyond $1 \mathrm{MHz}$, it is necessary to employ undersampling techniques as described by Hartov et al. (2000). To minimize errors from the DSP algorithms, the sample rate must be controlled to at least 1-Hz accuracy. In the LCT2, this is achieved by controlling the timing of individual samples using the AD9833 DDS chip from Analog Devices, which is able to output a square wave of programmable frequency to a resolution of 0.1-Hz. Logic signals are produced using an Altera MAX 7000S programmable logic device, which is controlled from software via the USB interface and the 8051 microcontroller. The MAX 7000S is 3.3-V input voltage compatible, suitable for interfacing with the FX2 chip, and 5-V output voltage compatible, suitable for driving the multiplexer, DDS and ADC. The chip is reprogrammable and, as it is based on EPROM technology, the data are non-volatile. The Quartus II software, required to verify the design and program the device, is freely available from the Altera website (www.altera.com). A photograph of the LCT2 instrument is shown in Figure 6.

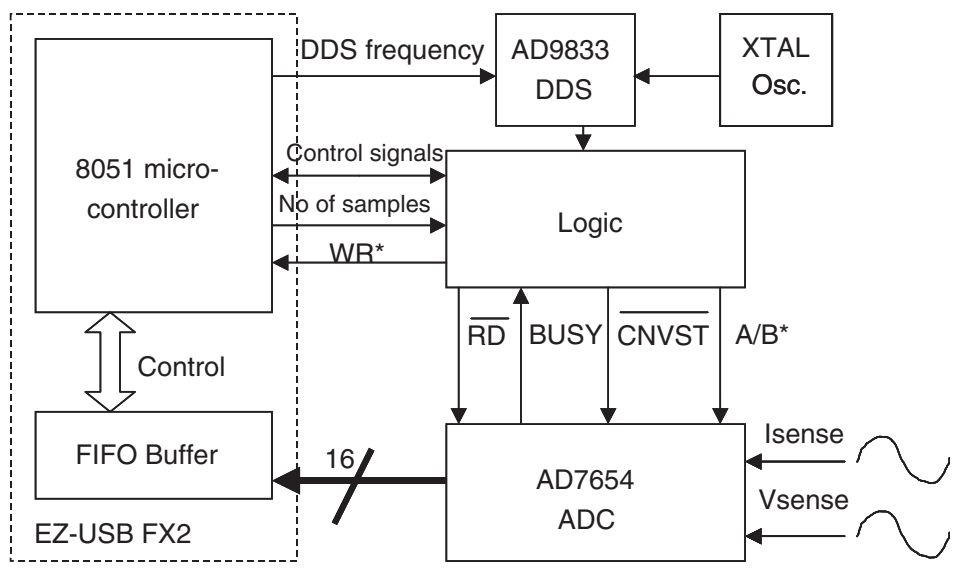

Figure 5 Data acquisition module

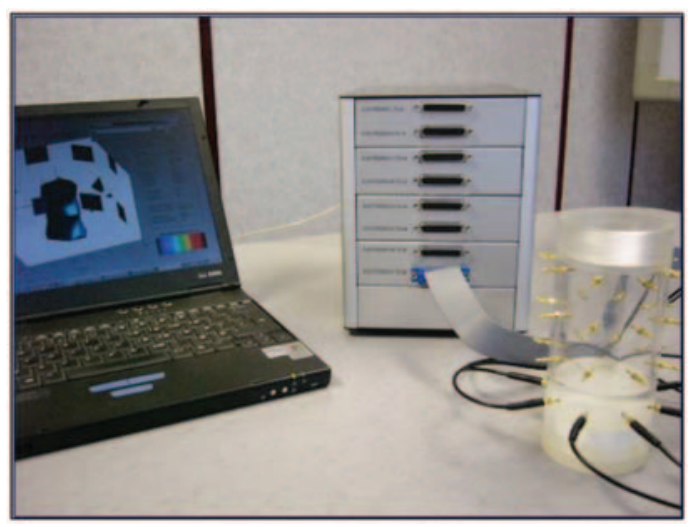

Figure 6 The LCT2 
Signals are routed between the SCB and the electrodes by a multiplexer in the form of a cross-bar switch in a similar way to that described by Yerworth et al. (2002). Instructions for configuring the multiplexer are sent from the 8051 microcontroller via the Altera MAX. The multiplexer can be re-configured in about $1 \mathrm{~ms}$. Importantly, in the context of accessibility, this multiplexer architecture is selected in order to offer maximum versatility in choosing measurement strategies. It is not restricted to the popular adjacent strategy and includes the possibility of exciting or measuring on multiple electrodes. Each multiplexer card serves eight electrodes and the basic configuration of the LCT2 includes eight cards, implemented as daughterboards that plug into the motherboard comprising USB interface and signal conditioning board.

\section{LCT analyser control software architecture}

The LCT instrument's software architecture has been structured into three distinct sections, as illustrated in Figure 7. The purpose of this is to allow the common core control code to be readily interfaced with alternative reconstruction algorithms and instrumentation firmware or hardware, as these are developed by tomography research groups.

\subsection{Core LCT package}

The core package allows a basic 3D finite element mesh (FEM) structure to be configured as a system model. The term system model may be defined as the mesh representation of the subject body, or process, in conjunction with the associated matrices to identify the electrode structures, drive protocol and, if appropriate, image

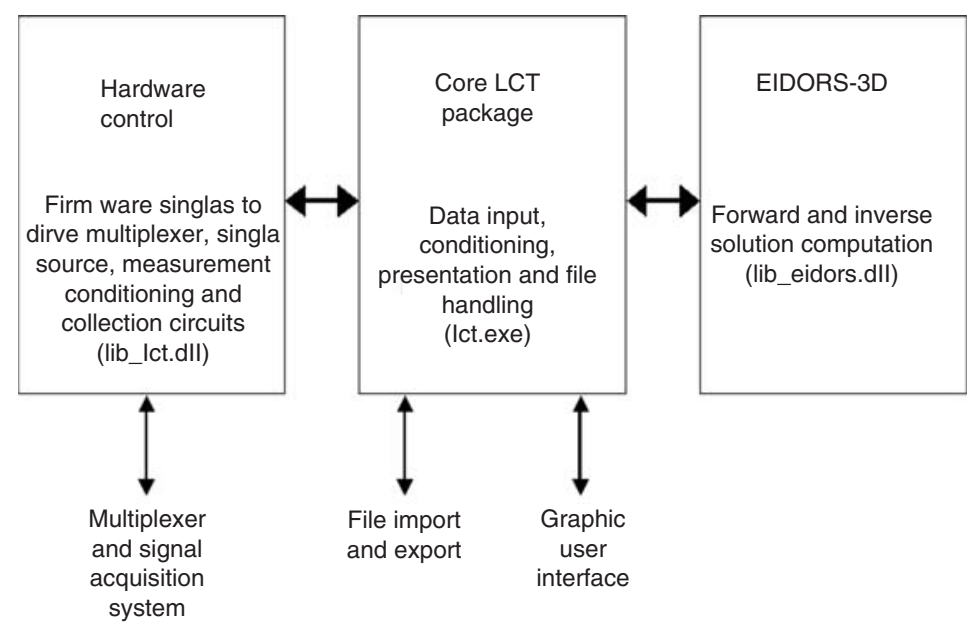

Figure 7 The LCT control panel software architecture 
reconstruction parameters. The FEM typically is automatically created from primitives and imported as a text file from a package such as Netgen (www.hpfem.jku.at/ netgen). As the theoretical forward solution is based upon a user-defined reference material matrix, inaccuracies in mesh design or electrode contact impedance can result in an imprecise Jacobian and poor measurement prediction. Because of this, the LCT Control Panel allows for both an absolute or relative calibration. The latter utilizes the inverse solution, as computed for a subject body or process having known electrical characteristics, to adjust the reference material matrix and, in the case of an iterative solution, the Jacobian as well. In addition to configuring the system models, the core package also handles the 2D measured signal plots and 3D depictions of the structural model (Figure 8) or the reconstructed images (Figure 9). The latter may be displayed as static or scanned, single or multiple, cuts through the body or as rendered images, in the form of isofaces, contour plots or real/imaginary surface plots. Any of these displays may then be created into a movie file, by the Control Panel, and viewed externally using a media player compatible the MPEG4 codec, as typically used by digital video disc players.

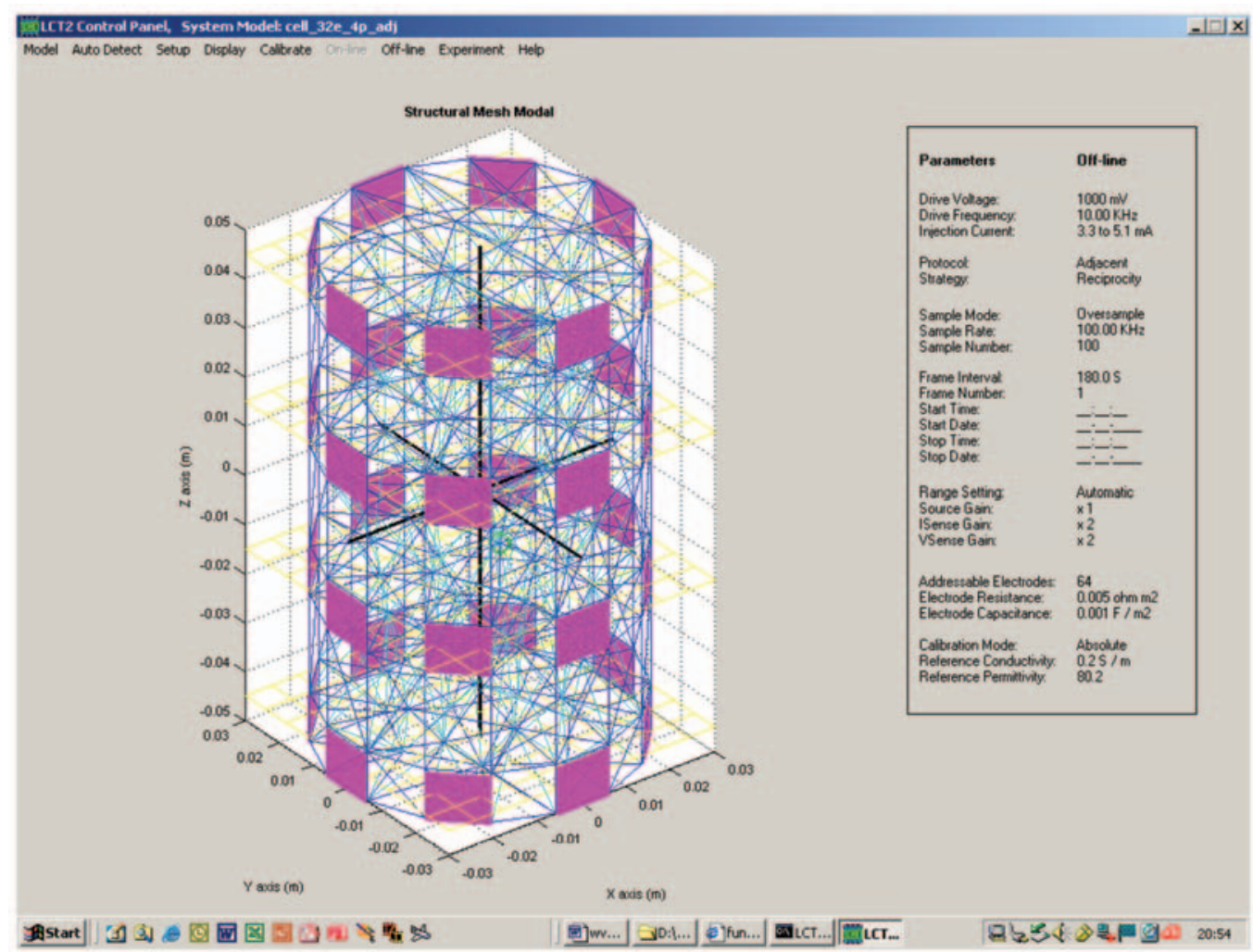

Figure 8 32-Electrode system model 


\subsection{EIDORS-3D library}

To act as a platform to develop the EIDORS-3D project against practical datasets, all the EIDORS functions used within the package have been drawn together into one dynamically linked library, with the title lib_eidors.dll. This contains all the necessary algorithms, 11 in total, to generate the forward, linear-inverse and fixed step iterativeinverse solutions. The default library being based upon the EIDORS-3D, version 2.0, functions (Polydorides and Lionheart, 2002). Provided the input arguments and returned variables are configured as per the original library, these functions may be readily re-written by tomography researchers and 'plugged' back into the core package to study the effects of novel image reconstruction techniques. By adopting the EIDORS-3D approach, the LCT Control Panel offers the non-specialist user, who wishes to use electrical impedance tomography (EIT) as a tool to develop their own discipline, direct access to the powerful model analysis capabilities offered by singular value decomposition (Lionheart, 2004), combined with the ability to quickly and intuitively view the effect of alternative electrode and current pattern arrangements (Figures 10 and 11). The latter may be configured automatically, for

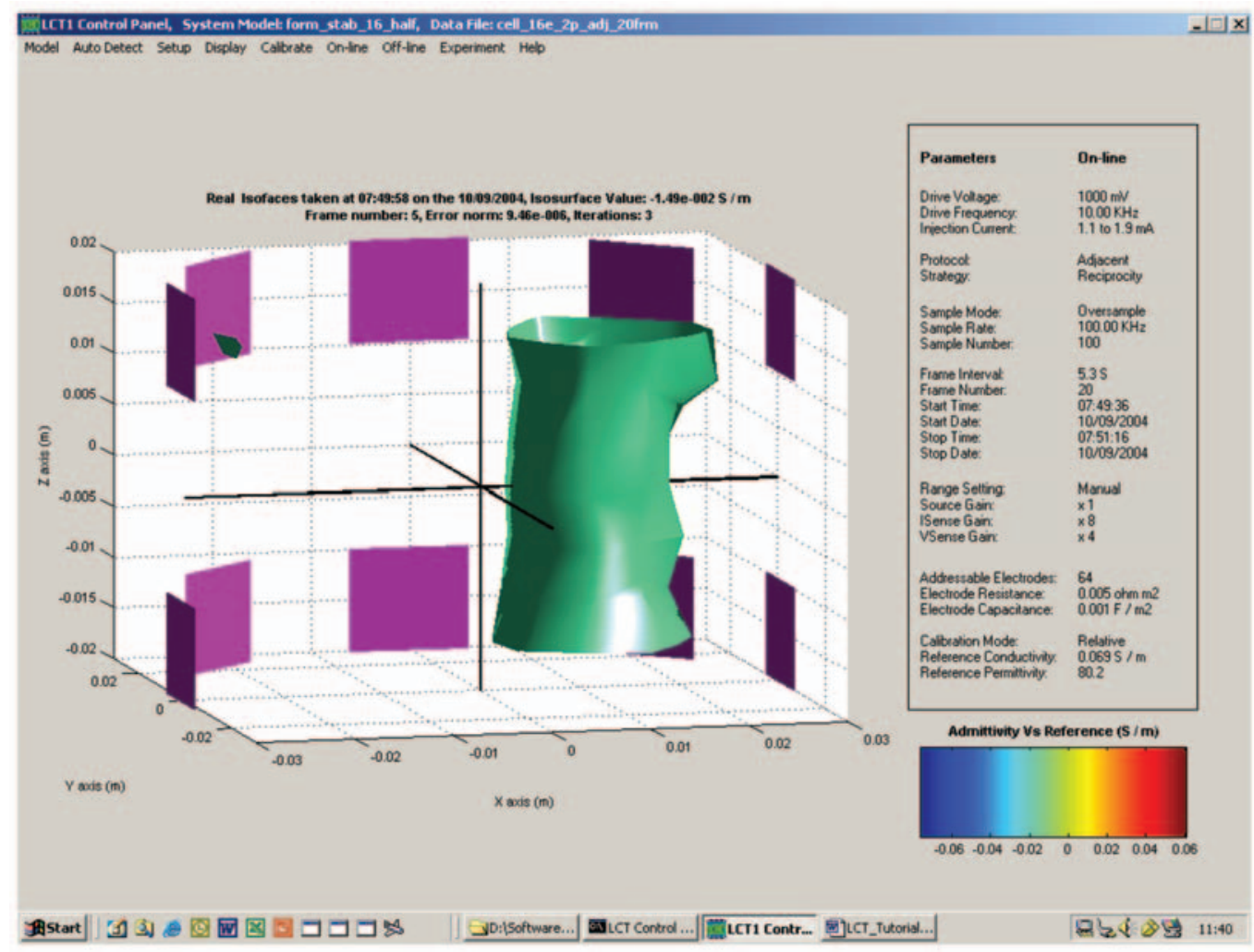

Figure 9 3D photo-real rendered isofaces 


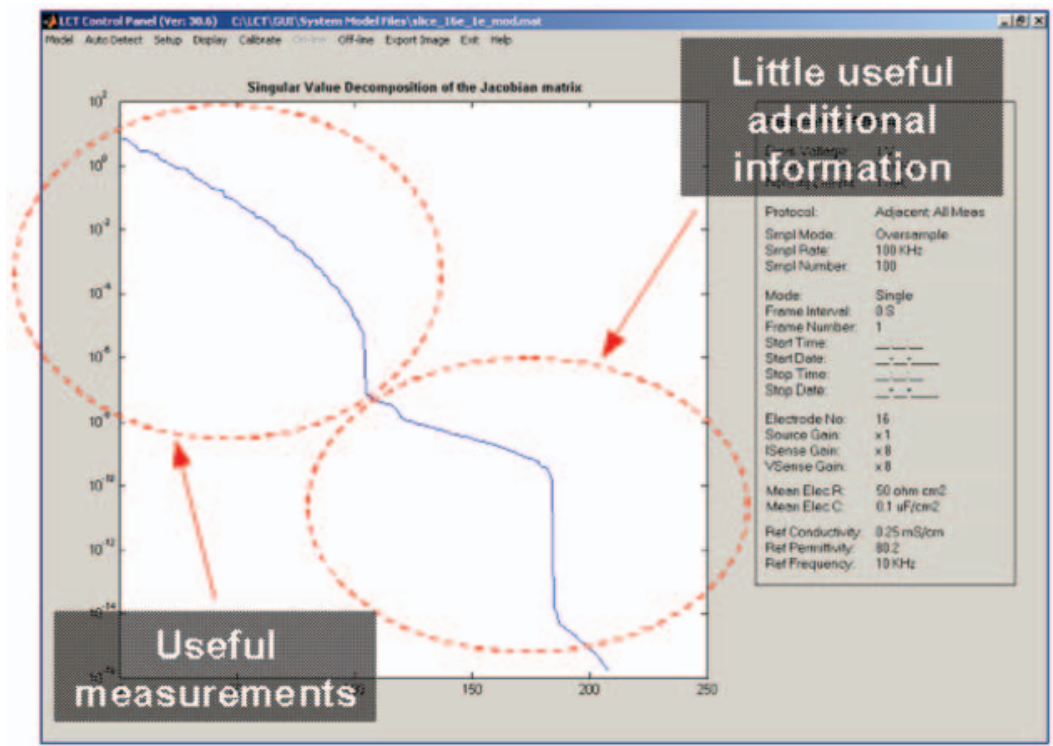

Figure 10 SVD analysis of system model

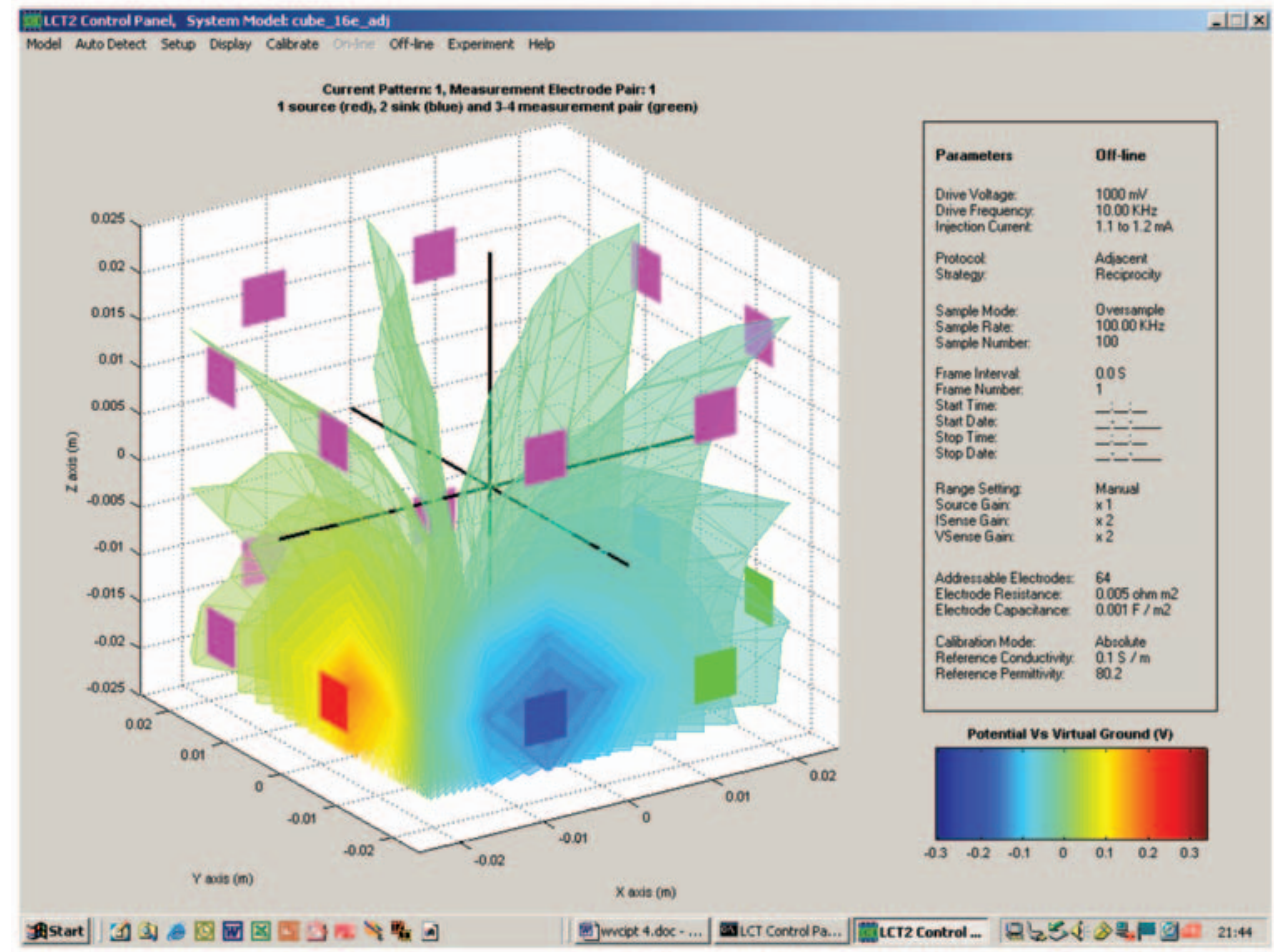

Figure 11 Forward solution for a 'cube' model 
the classic adjacent or opposite strategies, or imported from a text file, to examine custom drive protocols.

\subsection{Hardware control library}

In a similar fashion to the EIDORS library, the hardware and firmware handling functions for the LCT instruments are held within one common gateway library, under the title lib_lct.dll. This may be 'unplugged' and exchanged to reconfigure the system for the LCT2 or any future hardware manifestation. With the advent of the secondgeneration instrument, a standardized series of six, $\mathrm{C}++$ language, interface functions have been defined, covering the various aspects of data collection, calibration and initialization of the firmware. These six functions may then be used by electronic and software engineers as the entry point to subsidiary libraries specific to the needs of their future instrumentation hardware.

\section{Experimental testing}

Characterizing an instrument is important in determining the confidence levels in any reconstruction performed using its measurements. Information concerning the instrument's electronic performance is useful, but results obtained from tomographic situations are more pertinent, since they incorporate realistic loading conditions. This is of particular relevance to the LCT instrument since the performance of its voltage-sourcing output stage can be influenced by the impedance of any connected load. Therefore, measurements were acquired from a tomographic vessel using several aqueous solutions of varying conductivities. The vessel used for the following results was a cylindrical acrylic container with a diameter of $200 \mathrm{~mm}$ and a height of $140 \mathrm{~mm}$. Thirty-two silver-plated copper disc electrodes were evenly spaced around the periphery forming two rings of 16 electrodes (Figure 12). The electrode array occupied around one-third of the available circumference per ring with each plane being $100 \mathrm{~mm}$ apart. Silver-plating of the electrodes was adopted to reduce the boundary impedance at the sensor interface. This was then modelled in the LCT instruments control software as a fixed series impedance. Though this is not a complete model of the electrode boundary conditions, it provides adequate accuracy, at any one selected frequency, to enable impedance imaging with this type of measurement cell arrangement. When modelling the vessel structure, the electrodes were depicted as rectangular rather than disc elements to reduce the complexity of the FEM mesh, ie, a 393 volume element model was used to reconstruct the bulk within a single plane 16-electrode system (Figure 13). Despite the investigation, as described below, being based upon a single horizontal plane of rectangular electrodes, the data reconstruction allowed for the effects of the outof-plane current paths. 


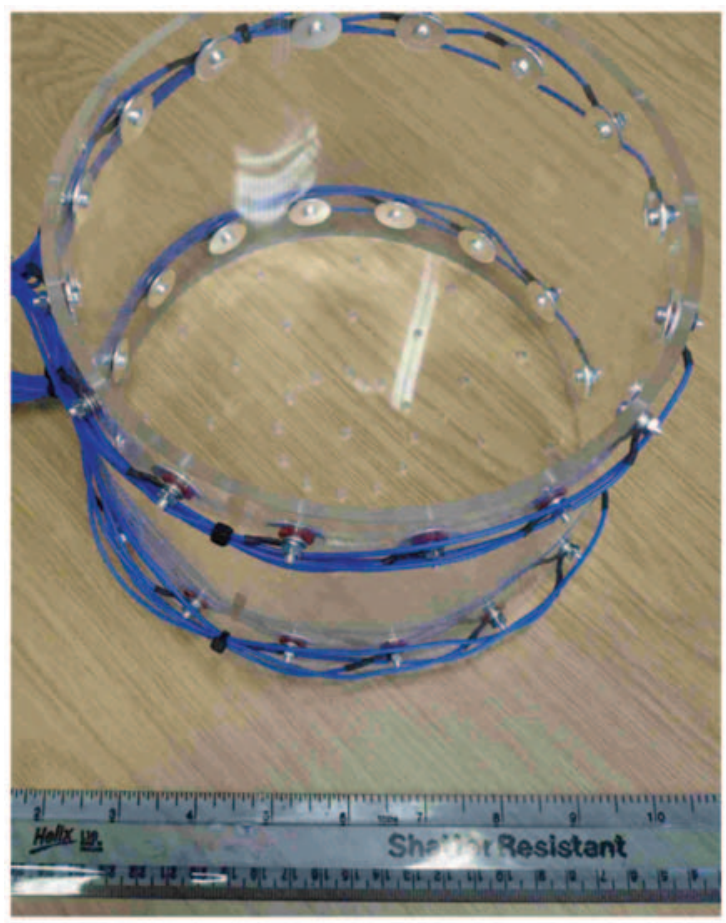

Figure 12 32-Electrode test cell

\subsection{Real and imaginary imaging of nylon and copper rods}

The LCT instrument was connected to the upper 16-electrode rings in the acrylic cell and two 200-mm long by 20-mm diameter vertical rods (phantoms) located $10 \mathrm{~mm}$ in from the vessel wall on either side of the cell, the left-hand one being nylon and the right-hand copper. A standard 'adjacent' strategy was then used to drive the electrodes at $500 \mathrm{mV}$ peak-peak amplitude and $10 \mathrm{kHz}$ frequency. The data returned by the LCT instrument was then recorded versus a reference homogenous salt/water solution having a conductivity of $0.1 \mathrm{~S} / \mathrm{m}$ and $\varepsilon \mathrm{r}$ of 80.2 (water). The corresponding real (in-phase) and imaginary (normal-phase) tomogrammes were then reconstructed as illustrated by Figures 14 and 15.

These images indicate that the instrument is capable of imaging and analysing features that may be optimally detected, as a function of frequency, at phase angles other than $0^{\circ}$ or $90^{\circ}$ from the drive signal. To further illustrate this point, Figures 16 and 17 depict the raw returned amplitude and phase measurements upon which the previous images were reconstructed, the blue and red lines in each graph indicating the measured signal versus the reference model's prediction in each case. The reconstruction is based upon the iterative Newton-Raphson solution (Polydorides and Lionheart, 2002). 


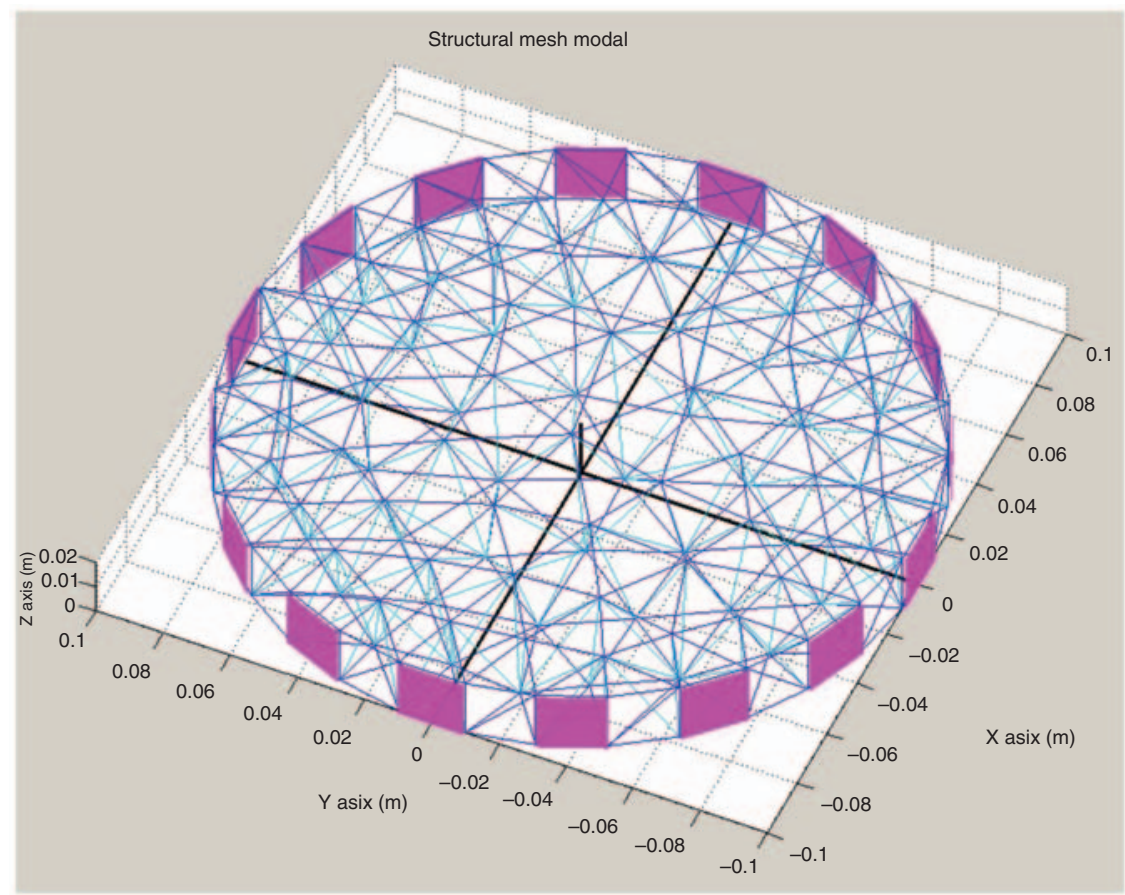

Figure 13 16-Electrode model of test cell upper ring

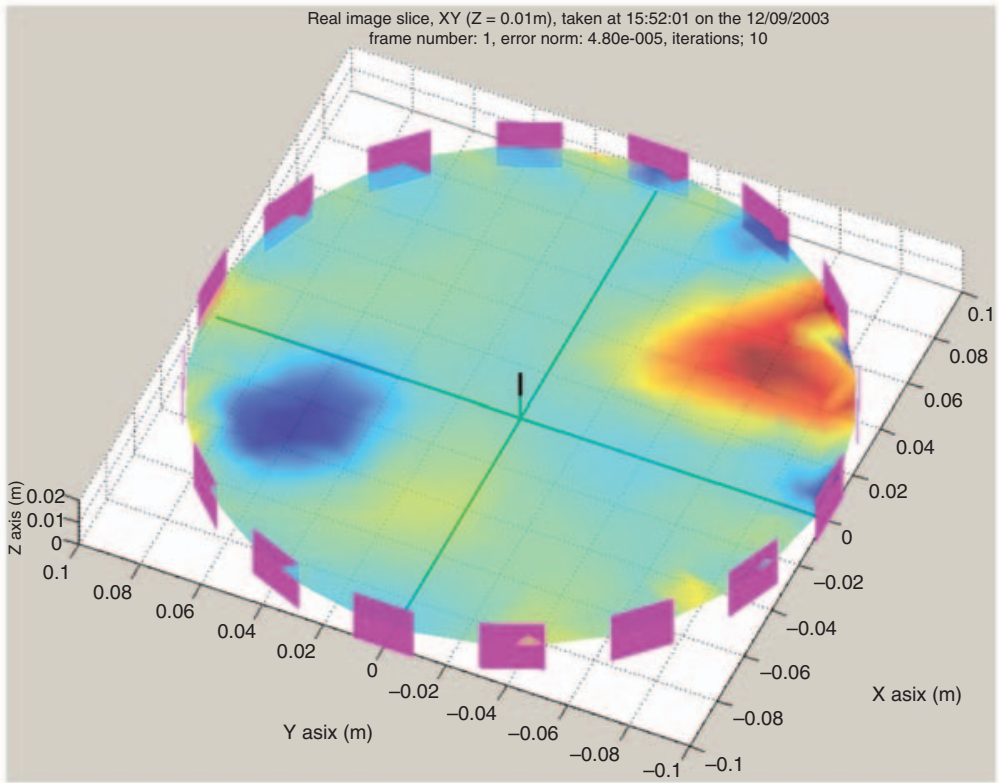

Figure 14 Real image of phantoms 


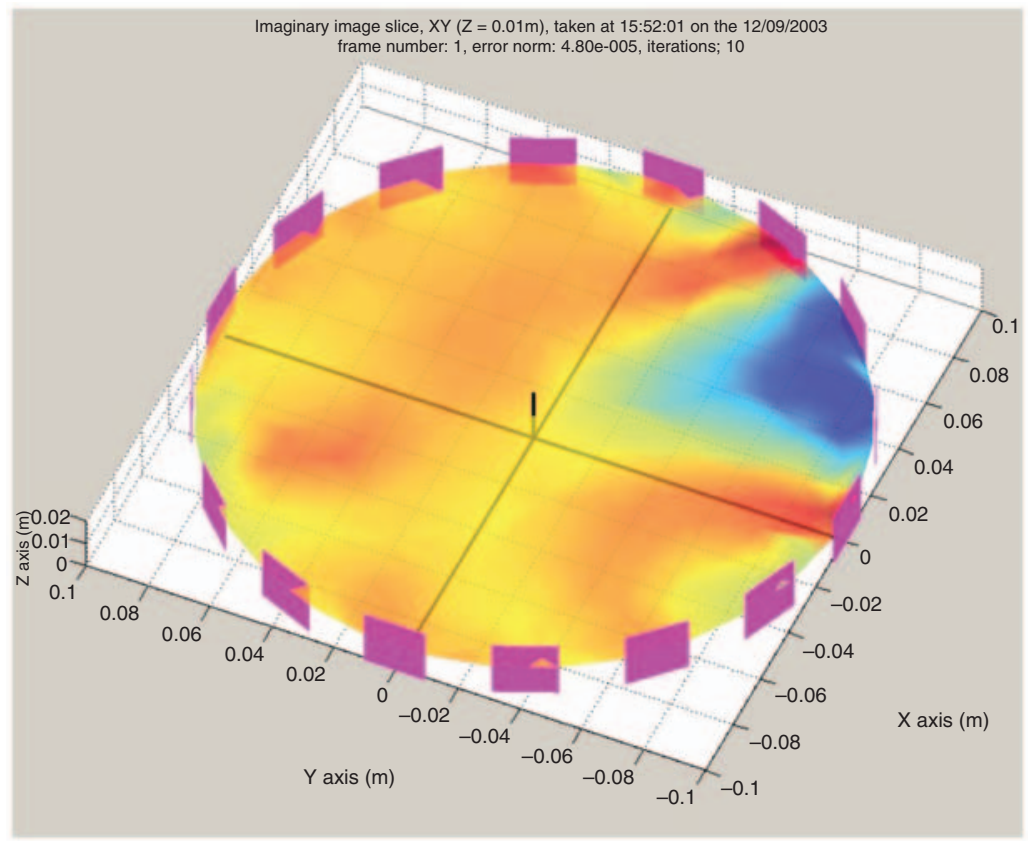

Figure 15 Imaginary image of phantoms

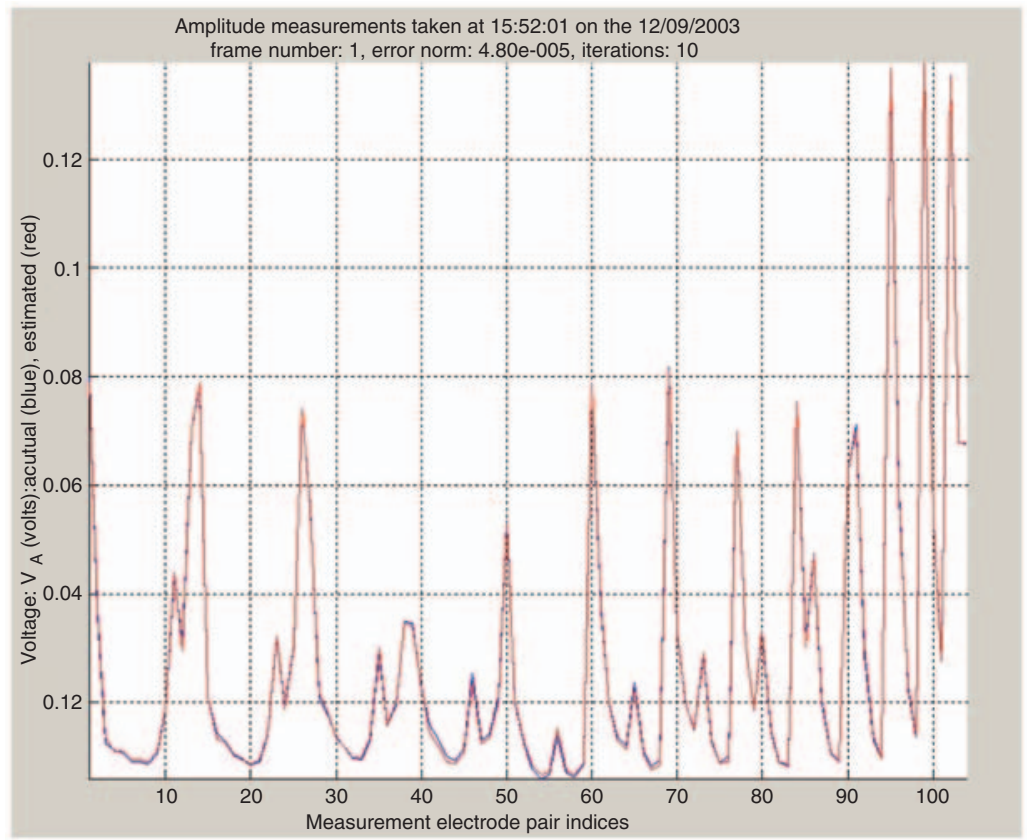

Figure 16 Amplitude measurements 


\subsection{Instrument accuracy analysis}

For the 16-electrode cell described above, 100 frames of an adjacent strategy (204 measurements) were acquired and the standard deviations were calculated for each complex admittivity measurement made on a series of brine solutions of varying concentrations. An arbitrary stimulation frequency of $10 \mathrm{kHz}$ was used for these experiments. Tables 1 and 2 display the maximum and mean deviations as a percentage of measured real and imaginary voltage signals respectively for four solutions.

The varying degrees of performance for different admittivities of solution are related to the corresponding signal strength degradation. The performance of the LCT

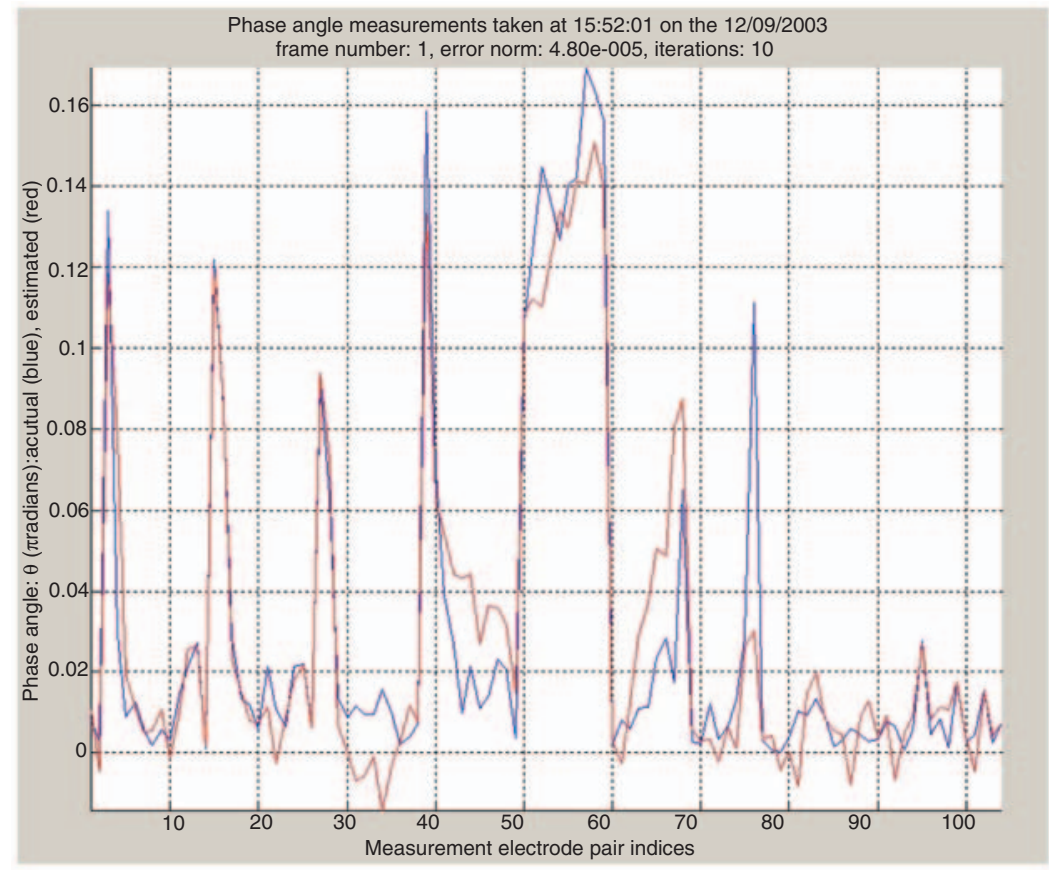

Figure 17 Phase angle measurements

Table 1 Real (resistance) signal analysis

\begin{tabular}{lllll}
\hline $\begin{array}{l}\text { Real/in-phase } \\
\text { admittivity }(\mu \mathrm{S} / \mathrm{cm})\end{array}$ & $\begin{array}{l}\text { Average } \\
\text { deviation }(\%)\end{array}$ & $\begin{array}{l}\text { Average } \\
\text { SNR }(\mathrm{dB})\end{array}$ & $\begin{array}{l}\text { Maximum } \\
\text { deviation }(\%)\end{array}$ & $\begin{array}{l}\text { Worst } \\
\text { SNR }(\mathrm{dB})\end{array}$ \\
\hline 5.8 & 0.24 & 52.3 & 1.19 & 38.5 \\
119 & 0.14 & 58.4 & 0.30 & 50.6 \\
3.72 & 0.17 & 55.2 & 0.60 & 44.5 \\
13.6 & 0.46 & 46.7 & 1.64 & 35.7 \\
\hline
\end{tabular}

SNR, signal-to-noise ratio. 
instrument with very highly conductive solutions is ultimately determined by providing a measurable current from the 10-bit DDS used to generate the output signals. Other techniques will improve performance such as adjusting stimulation frequency, output current and levels of measurement amplification, and they are all easily manipulated via the control software with no requirement for additional calibration. As a result, the LCT instrument, in conjunction with the EIDORS-3D algorithms, appears to be a useful research tool for investigating applications where full impedance information at multiple frequencies can provide additional insights over the more traditional in-phase (resistance)- or normal-phase (capacitance)-only measurements. It is unique in offering totally flexible excitation strategies for process tomography via the use of a cross-bar switch that allows any pairs of electrodes to be used as a source of current or for differential voltage measurement.

The results indicate that the greatest precision is obtained when the solution is predominately conductive but with bulk impedance greater than the LCT2 instrument's output impedance. Measurements of highly conductive materials will be dominated by electrode contact impedance effects, whereas low conductive materials are dominated by capacitive errors particularly those associated with cabling. Measuring these extremes accurately is difficult but difference techniques and more advanced calibration can make these experiments useful.

\section{Implementation against fine chemicals processing}

As stated earlier, the LCT project has been focused towards creating an analyser for investigating processes with relatively modest dynamics, as opposed to the more traditional higher-speed applications for capacitance or resistance tomography instruments, eg, multi-phase flow, combustion monitoring, mixing studies, pneumatic conveyance imaging, etc. A pertinent exemplar of the lower frame-rate duties is the analysis of formulations stability for speciality chemicals. Here a multi-phase product may be required to have a shelf life of several months or years without suffering permanent degradation, say only requiring mild agitation prior to eventual use. Though ostensibly inferential, low frame rate EIT instrumentation can

Table 2 Imaginary (dielectric) signal analysis

\begin{tabular}{lllll}
\hline $\begin{array}{l}\text { Imaginary/normal-phase } \\
\text { admittivity }(\mu \mathrm{S} / \mathrm{cm})\end{array}$ & $\begin{array}{l}\text { Average } \\
\text { deviation }(\%)\end{array}$ & $\begin{array}{l}\text { Average } \\
\text { SNR }(\mathrm{dB})\end{array}$ & $\begin{array}{l}\text { Maximum } \\
\text { deviation }(\%)\end{array}$ & $\begin{array}{l}\text { Worse } \\
\text { SNR }(\mathrm{dB})\end{array}$ \\
\hline 5.8 & 0.22 & 53.1 & 0.43 & 47.3 \\
119 & 0.75 & 42.5 & 7.07 & 23.2 \\
3.72 & 1.72 & 35.3 & 10.84 & 19.3 \\
13.6 & 1.28 & 37.9 & 3.62 & 28.8 \\
\hline
\end{tabular}

SNR, signal-to-noise ratio. 
provide new insight into the heterogeneous kinetics and chemical mechanisms occurring during ageing of a formulated product and so help to design the appropriate stabilizers and active ingredient combinations to give a product the required longer-term stability.

Changes in the continuous phase of a formulation will have a direct impact on the impedance spectra from the EIT instrument, whereas variations in any discontinuous phase or suspended solids will affect the permittivity as well as indirectly altering the conductivity. The deconvolution of this data into chemically meaningful information requires close cooperation between electrical tomography and formulations scientist. However, as an indication of failure of a given formulation recipe, 3D EIT's capability to detect loss of homogeneity, in a non-destructive and non-intrusive manner, can provide a screening tool for formulation recipe failure, which is readily compatible with high throughput laboratories.

The sensor that has been used for this work is shown in Figure 18. The sensor electrodes are fabricated as flexible printed circuit boards that are attached as eight vertical strips on the inside of the beaker. The electrodes are gold-plated and connections are taken out to the LCT using a 20-way FFC socket. The vertical

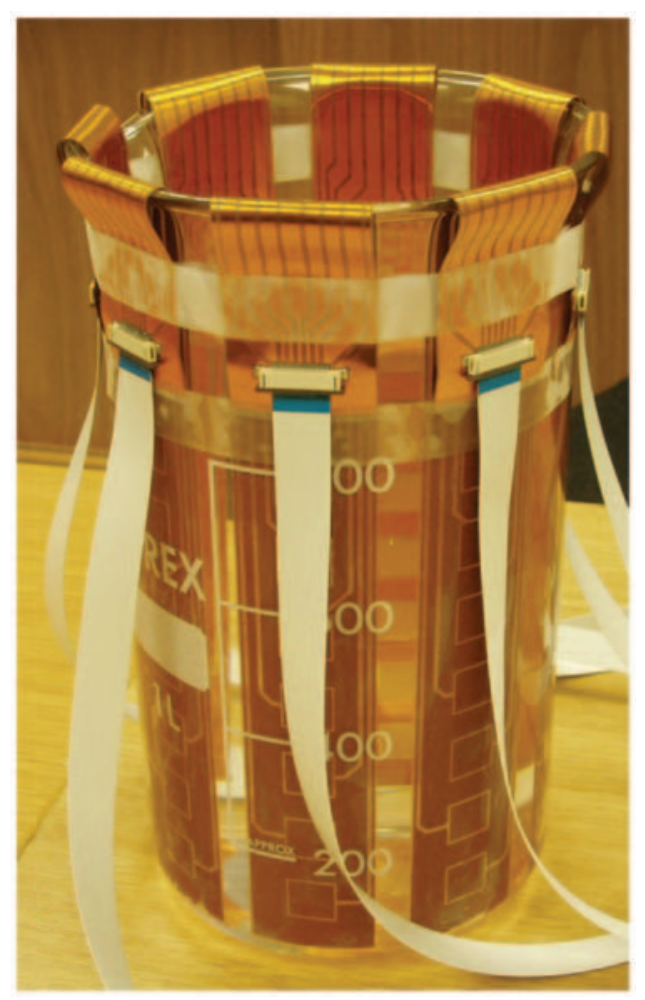

Figure 18 Formulation stability sensor vessel 
strips each include eight electrodes resulting in a total of 64 electrodes. Extensive modelling of the vessel was undertaken by FEM to facilitate subsequent image reconstruction. Vessel modelling suggested that the accuracy of sensor placement need only be within a few per cent of the vessel diameter. Vessel construction uses a combination of non-corrosive silicone sealant and pressure sensitive adhesive tape to mount the flexi-PCBs firmly on the glass wall. Generally, the vessels performed well and in the event of a sensor failure, it was a straightforward task to replace a single flexi-PCB strip.

During accelerated ageing trials, frame rates of several minutes, hours or even days may be appropriate to track the onset of instability. Rather than attempting to freeze rapid features, in this form of EIT it is often necessary to compress the time axes so that slowly varying characteristics may be identified. This may be illustrated with reference to Figure 19, which show a series of tomograms taken through the centre line, or Z-axis, of a formulations test vessel, in the $X Z$ and $Y Z$ orthogonal planes. The arbitrary scale being shown underneath with the lowest to highest admittivity values being shown from blue to red. These represent a 4-day experiment, where

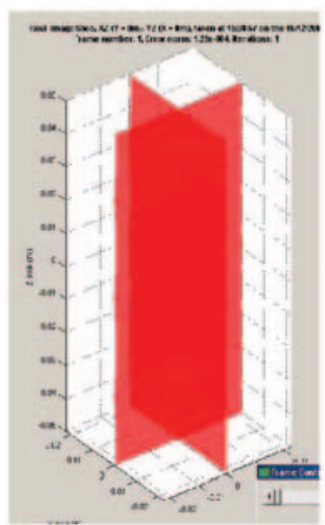

(a)

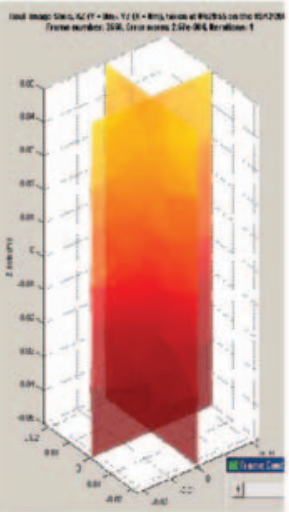

(b)

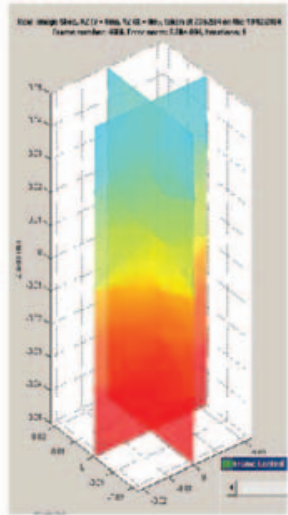

(c)

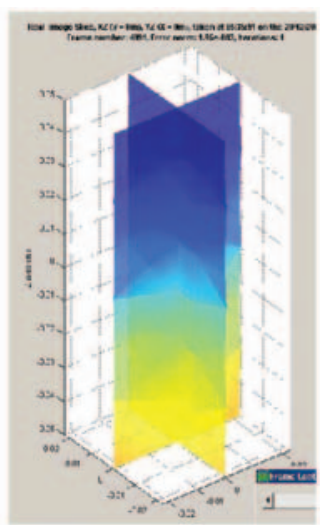

(d)

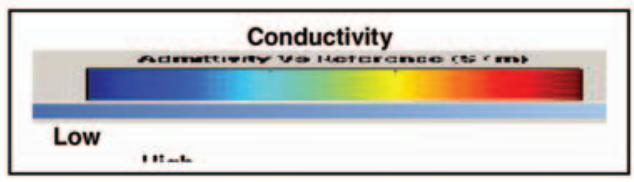

Figure 19 Reconstructed images of formulation stability: (a) homogeneous $(t=0 \mathrm{~h})$; (b) sedimentation $(t=60 \mathrm{~h})$; (c) sedimentation $(t=75 \mathrm{~h})$; (d) rapid breakdown $(t=80 \mathrm{~h})$ 
Figure 19(a) is the homogeneous initial condition of the sample. In all cases, an adjacent excitation strategy was utilized. The subject material, in common with many formulations, was visually opaque and contained significant solids loading, which gave it an extinction coefficient that was incompatible with optical spectroscopies, such as near-infrared or ultraviolet.

After an initial period of stability, around $60 \mathrm{~h}$, the EIT image in Figure 19(b) shows that the sample starts to sediment. This process then progresses for around half a day, as represented in Figure 19(c), until a relatively rapid phenomenon occurs, over about a 90-min period, and the highly conductive lower layer is seen to be exchanged for a lower conducting series of strata. This process is fully established after $80 \mathrm{~h}$, as shown in Figure 19(d). The reasons behind this particular rapid collapse in stability may be speculated upon, eg, a full or partial phase inversion resulting from a gradual continuous reaction mechanism occurring elsewhere in the sample. Of significance here is the use of the LCT to detect, in real-time, the onset of breakdown.

\section{Summary}

A new electrical impedance tomography system that has been designed with the intention of facilitating accessibility to the technology is described. Accessibility is provided in the form of: low cost; flexibility in considering electrode geometries; measurement strategies; excitation frequencies; availability of data files; freely available software for mesh generation, modelling and image reconstruction. The innovation in this instrument is based upon the combining these features into one small footprint instrument, which may then create a complete tomograph when connected to the USB port of a conventional laptop. The system provides a measured, differential current input and receives the measured differential voltages that are amplified and then digitized. Amplitude and phase are recovered in software using digital signal processing algorithms to give true impedance data. The second version of the instrument, the LCT2, has been described in this paper and replaces the earlier DAQ card interface with a USB2 link and a bespoke data acquisition based on a 16-bit ADC with under- or oversampling. The control panel interfaces the hardware to the EIDORS 3D software. 3D mesh information can be loaded from programs such as NETGEN and configured to provide a full model including measurement strategy and material properties. Results are available as 3D images, iso-surfaces, movies or raw data. The instrument can provide 16-electrode measurement frames in about $1 \mathrm{~s}$ and is intended to support impedance tomography research and act as a platform for the EIDORS-3D suite of reconstruction algorithms. It has been analysed against water samples of varying salt content $(0.01-2 \mathrm{mS} / \mathrm{cm}$ conductivity, $\varepsilon \mathrm{r}=80.2)$ containing both conducting and non-conducting phantoms. 


\section{References}

Bottomley, P.A., Rogers, H.H. and Foster, T.H. 1986: NMR imaging shows water distribution and transport in plant root systems in situ. Proceedings of the National Academy of Sciences USA 83, 87-9.

Davidson, J.L., Ruffino, L.S., Stephenson, D.R., York, T.A. and Grieve, B.D. 2004: 3 dimensional electrical impedance tomography applied to a metal-walled filtration test platform. Measurement Science and Technology $15,2263-74$.

Grieve, B.D., Davidson, J.L., Mann, R., Lionheart, W.R.B. and York, T.A. 2003: Process compliant electrical impedance tomography for wide-scale exploitation on industrial vessels. 3rd World Congress on Industrial Process Tomography, Banff, 2-5 September, 806-12.

Hartov, A., Mazzarese, A., Reiss, F.R., Kerner, T.E., Sunshine Osterman, K., Williams, D.B. and Paulsen, K.D. 2000: A multichannel continuously selectable multifrequency electrical impedance spectroscopy measurement system. IEEE Transactions on Biomedical Engineering 47, 49-58.

Hunt, A., Pendleton, J.D. and White, R.B. 2003: A novel tomographic flow analysis system. 3rd World Congress on Industrial Process Tomography Banff2-5 September, 281-86.

Jaworski, A.J. and Dyakowski, T. 2001: Application of electrical capacitance tomography for measurement of gas-solids flow characteristics in a pneumatic conveying system. Measurement Science and Technology 12, 1109-19.

Lionheart, W.R.B. 2004: EIT reconstruction algorithms: pitfalls, challenges and recent developments. Physiological Measurement 25, $125-42$.
Ma, X., Peyton, A.J., Binns, R. and Higson, S.R. 2003: Imaging the flow profile of molten steel through a submerged pouring nozzle. 3rd World Congress on Industrial Process Tomography, Banff, 2-5 September, 736-42.

Polydorides, N. and Lionheart, W.R.B. 2002: A MATLAB toolkit for three-dimensional electrical impedance and diffuse optical reconstruction software project. Measurement Science and Technology 13, 1871-83.

Ricard, F., Brechtelsbauer, C., Lawrence, C., Xu, Y. and Pannier, A. 2003: Application of electrical resistance tomography technology to pharmaceutical processes. 3rd World Congress on Industrial Process Tomography, Banff, 2-5 September, 701-706.

Wilkinson, A.J, Randall, E.W, Cilliers, J.J., Durrett, D.R., Naidoo, T. and Long, T. 2005: A 1000-measurement frames/second ERT data capture system with real-time visualization. IEEE Sensors Journal 5, 300-307.

Yerworth, R.J, Bayford, R.H, Cusick, G, Conway, $\mathbf{M}$ and Holder, D.S 2002: Design and performance of the UCLH Mark 1b 64 channel Electrical Impedance Tomography (EIT) system, optimized for imaging brain function. Physiological Measurement 23, 149-58.

York, T.A., Burnett-Thompson, A. and Grieve, B.D. 2004: Low cost and accessible electrical impedance imaging. 3rd International Symposium on Process Tomography in Poland, Łodz, September, 175-78.

York, T.A. 2005: Electrical tomography for industrial applications. In David Holder, editor. Electrical impedance tomography: methods, history and applications. IOP series in Medical Physics and Biomedical Engineering. University College, 295-347. 